\title{
Molecular epidemiology of $C$. pneumoniae infections
}

\author{
Alisa Shurdhi', Federica Delucca', Francesca Savegnago', Maria Di Paolo', Manuela Donati' \\ I Sezione Microbiologia Policlinico S. Orsola - Malpighi via Massarenti 9, 40138 Bologna
}

Key words: Chlamydophila pneumoniae, respiratory diseases, RT-PCR

Epidemiologia molecolare delle infezioni respiratorie da Chlamydophila pneumoniae

\section{SUMMARY}

Introduction. Chlamydophila pneumoniae (C. pneumoniae) is one of the most common respiratory pathogen, with an incidence of infection varying from $6 \%$ to $20 \%$. The present study aimed to assess the incidence of $C$. pneumoniae infections in patients with acute respiratory diseases using a RealTime PCR (RT-PCR) method.

Methods. In the period January 2007-December 2008279 biological samples coming from patients (I 90 males and 89 females) with acute respiratory infections was collected and tested. Samples have been extracted using NucliSens easyMag Biomerieu according to manufacturer's instructions and amplified by LightCycler Real-Time PCR Roche for the detection of C. pneumoniae DNA.

Results. Data analysis revealed a higher prevalence of C. pneumoniae infections in male patients (7.9\%) than in females $(5.6 \%)$. In addition, it is interesting to note that the incidence of $C$. pneumoniae infection was higher $28.6 \%$ in the period February - April.

Conclusions. The results obtained in this study confirm the utility of molecular techniques in laboratory diagnosis and epidemiological investigations of respiratory infection caused by $C$. pneumoniae. RT-PCR have proved to be a rapid and a reliable technique to monitor and treat opportunely C. pneumoniae infections to avoid short and medium/long term complications.

\section{INTRODUZIONE}

C. pneumoniae risulta essere tra i più comuni agenti eziologici di infezioni respiratorie, con una incidenza variabile dal 6 al 20\% (3). L'obiettivo di questo studio è stata la valutazione dell'incidenza di $C$. pneumoniae in pazienti con infezioni respiratorie acute mediante l'impiego della tecnica di biologia molecolare RealTime PCR (RT-PCR).

\section{METODI}

Nel periodo gennaio 2007-dicembre 2008 sono pervenuti nel nostro laboratorio 279 campioni biologici provenienti da 190 pazienti di sesso maschile e 89 di sesso femminile, ospedalizzati per affezioni respiratorie acute. I campioni, rappresentati da 117 aspirati faringo-nasali (AFN), 159 lavaggi bronco alveolari (BAL) e 3 espettorati, sono stati estratti utilizzando il kit NucliSens easyMag della bioMérieux e successivamente amplificati mediante tecnica RT-PCR, impiegando il kit Chlamydia pneumoniae LCSet per LightCycler della Roche. Il limite di rilevabilità nei campioni clinici è stato di 5 equivalent genomici/reazioni di PCR.

TECNICA LIGHTCYCLER REALTIME PCR ROCHE Il materiale estratto è stato amplificato con metodica RT-PCR utilizzando lo strumento LightCycler II con il kit Chlamydia pneumoniae LCSet (TIB molbiol per Roche) (1). La metodica prevede l'utilizzo di capillari di vetro all'interno dei quali vengono messi a contatto $15 \mu \mathrm{l}$ di master mix e $5 \mu \mathrm{l}$ di DNA estratto dal campione. La miscela di amplificazione è stata preparata aggiungendo a primers e probes l'enzima hot start Taq DNA polymerase presente nel LightCycler FastStart DNA Master PLUS Hybridization Probes (Roche). Durante la reazione un frammento genomico (target gene) dell'agente patogeno (polymorphic membrane protein $G$ family di 140bp) viene amplificato usando primers specifici e rilevato con la fluorescenza generata dalla coppia di sonde specifiche di ibridazione (Figura I). La rivelazione si basa sulla lettura in tempo reale della fluorescenza emessa dai prodotti di amplificazione, direttamente nel capillare con un limite di di rilevamento di 5 copie (equivalenti genomici)/reazione PCR nei campioni biologici per entrambi gli agenti. La lettura avviene ad ogni ciclo su sei canali di lettura: utilizzando sonde marcate con fluorofori diversi (es. LC-Red 640 )

\section{RISULTATI}

La prevalenza di C. pneumoniae nella popolazione studiata è risultata del 7.2\% (20/279). È stata evidenziata inoltre una maggiore prevalenza, pari al $7.9 \%$, nel sesso maschile (età media 32 anni) rispetto al 5.6\% nel sesso femminile (età media 31 anni). I campioni clinici provenivano nel 56.6\% (158/279) dei casi da Ospedali esterni e nel 43.4\% (121/279) da reparti pediatrici. L'infezione è risultata più frequente nel periodo compreso tra febbraio e aprile del 2007 e del 2008, con un incidenza del 28.6\% (7/20).

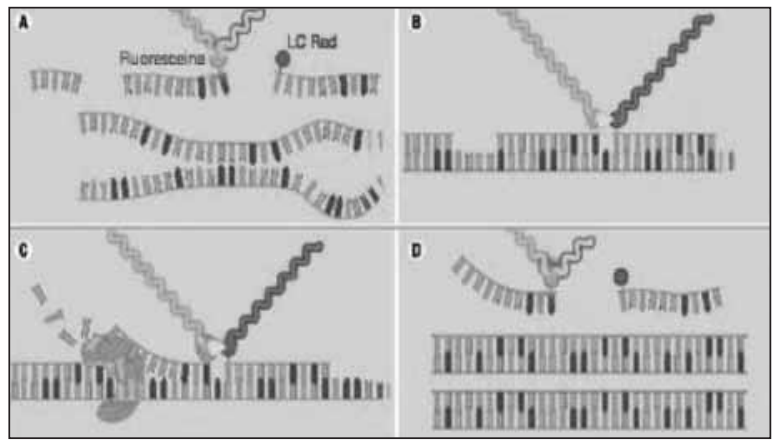

Figura I. II principio delle sonde FRET.

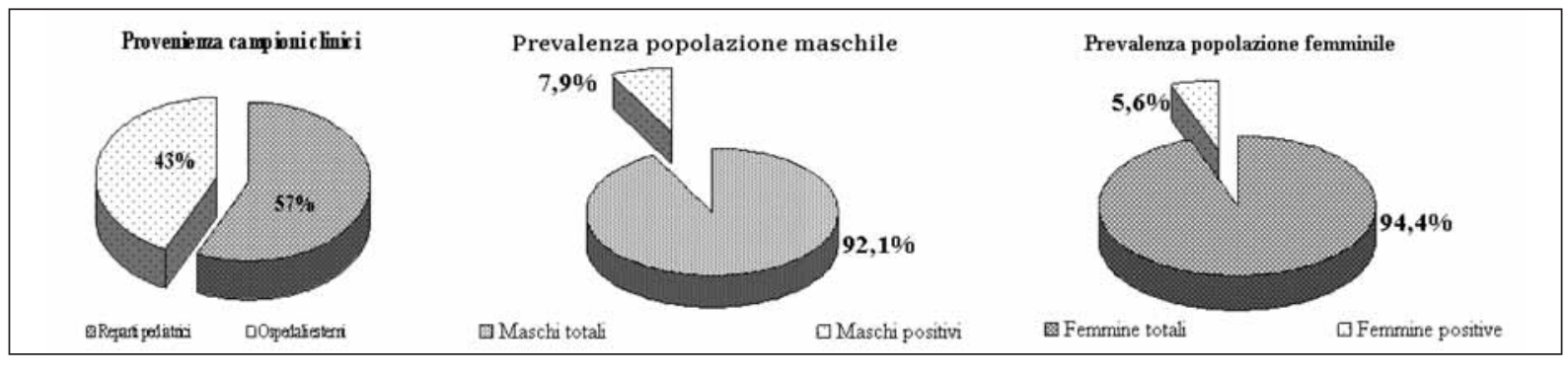

Corresponding author: Alisa Shurdhi

40138 Bologna - via Massarenti, 9

Tel. 05163645I3 - Fax 05/307397

E-mail: alisa_s@libero.it 


\section{CONCLUSIONI}

I dati ottenuti sono in linea con quelli descritti in letteratura (2) sia riguardo a distribuzione (maggiore nel sesso maschile) che a frequenza dell'infezione. La diagnosi e il monitoraggio epidemiologico dell'infezione da C. pneumoniae, attraverso tecniche di biologia molecolare, possono essere utili al clinico per rapidità di risposta e affidabilità del risultato. Essi sono rilevanti anche per la salute pubblica al fine di sorvegliare e curare tempestivamente eventuali gravi complicanze a breve termine (insufficienza multi-organo in pazienti immunode- pressi o clinicamente debilitati) e a medio-lungo termine (asma, BPCO e insufficienza respiratoria).

\section{BIBLIOGRAFIA}

1. Ciervo, Petrucca, Cassone, Ann Ig. 2003 Nov-Dec; 15(6): 851-61

2. Kurz H, Göpfrich H, Wabnegger L, Apfalter P. Pediatr Pulmonol. 2009 Sep;44(9):873-6.

3. Lieberman D, Shvartzman P, Lieberman D, Ben-Yaakov M, Lazarovich Z Hoffman S, Mosckovitz R,Ohana B, Leinonen M, Luffy D, Boldur I (1998). Etiology of respiratory tract infection in adults in a general practice setting. Eur J Clin Microbiol Infect Dis. Oct;17(10):685-9. 